\title{
From the Editors Desk: Angela Zawacki-Downing Writing to Professor Illana Gozes, Editor-in-Chief Journal of Molecular Neuroscience-Speaking from a Mother's Heart, AD's ADNP Syndrome
}

\author{
Angela Zawacki-Downing ${ }^{1}$
}

(C) Springer Science+Business Media, LLC, part of Springer Nature 2019

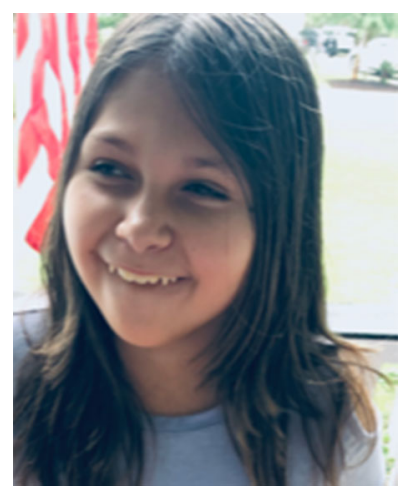

Our sweet and sassy AD (our middle of three beautiful children) suffers from an ultra-rare neurogene alteration (mutation) in a gene called ADNP: Activity-Dependent Neuroprotective Protein. The ADNP gene is a most crucial gene of brain development and is important for neurogenesis. A long eight and a half year journey which included extensive investigation and genetic testing lead us to the diagnosis. At age 8.5, we were lead to East Tennessee Children's Hospital where we sent AD and our (parent) blood in for advanced genetic testing known now as genome sequencing.

Shockingly, in the months we awaited the results of her sequencing (from Ambry Labs in CA), a rare, new genetic syndrome was discovered, the ADNP Syndrome also known as the Helsmoortel Van Der Aa Syndrome.

Angela Zawacki-Downing

Charleston, SC, USA

We received this surprising news the very day of her diagnosis. As I turned to see the social worker holding a box of tissues, a clinic nurse, and my Husband's look of shock as we were delivered the results of this "last hope" testing, I wasn't sure if I wanted to jump for joy in the faithfulness of an answer or cry of the fear of not knowing what was next. RARE. A word that rings stronger than the word ADNP. Like many parents to ADNP syndrome patients, the discovery of this new syndrome/disease was a miracle in our minds even if we were told that "day of diagnosis" that AD shared this disease with a mere ten other "known cases" in the entire world. What did this mean? This meant my daughter was more than rare. She had an orphan disease with very little known about it. We were told by the lab in CA that although her symptoms matched this novel disease, it was possible she was the only known case in the US at that point. Quickly after her diagnosis, another mother found me online. We connected and within weeks, there were a handful of other parents, all connected through a special ADNP website, founded by one of the mothers (Sandra Bedrosian-Sermone). After over 8 years of waiting and with the condition being so rare, this progress sounded amazing but with a handful of diagnosed children compared to the 329 million US population (https:// www.worldometers.info/world-population/us-population/), it is not as comforting as one might think. Either way, we were no longer alone.

Sandra founded the ADNP Kids Research Foundation. Over time, with more connection being made with other families online across the globe, everyone began to fully realize how underdiagnosed or misdiagnosed these ADNP children were. At that point, it became our mission to be sure all kids in the US, Europe, and in the rest of the world were properly diagnosed. Surprisingly enough, at that time, according to scientific publications, the projected ADNP syndrome prevalence was $0.17 \%$ of the all autism cases with mutations in the ADNP gene presented as one of the most prevalent known 
monogenetic causes of autism. In support of "genome sequencing" for proper diagnosis and "earliest of interventions," we continued to tell our children's stories in hopes the US will continue to see the need for this advanced, sophisticated genetic testing, especially in cases such as AD's, where other genetic tests could not have given us answers to the etiology of her multiple symptoms. For me, I became a mother advocate and sat on the ADNP board for 2 years. Over time, another ADNP mother, living in NY, was able to recruit an amazing team, the Seaver's Center/Mt. Sinai in NY, NY for a $\$ 200,000 /$ launch project. Either way, it was an amazing feeling to have a "medical home" per se as we listened to their proposals and how they hoped to help our children now and in the future.

So what is ADNP? Why should you support funding research, the first ever clinical trial and future science?

Choosing to help us and invest in treatments for ADNP means you have realized how the study of neuroscience affects all of us. We all age. The brain is the most crucial organ of our body/the control panel for our organs. AD suffered from severe sleep disorders, with occasional events where she stopped breathing as an infant and toddler. She had a complicated infancy, most noticeably slow development, autism coupled with muscle weakness causing severely delayed walking that required massive hours of rehabilitation. The developmental delays did not explain the endocrine/growth issues, required surgeries, and the most devastating - the loss of her voice. Autism was not the only reason she struggled and the neurological complexity suddenly coming to light with each parent/family connecting to the support group. AD also had a severe motor movement disorder - quoted by her physical therapist who worked with her $1-5$ years of age: "AD is still not baring weight independently (close to age 3 years) with intensive physical therapy (both land and water) her condition is mirroring traumatic brain injury and stroke patients I used to treat in geriatric therapy years ago." $\mathrm{AD}$ gained the ability to walk with an abnormal gait and a much slower rate when compared to her peers, as was stated in supplemental ADNP medical journal about significant gross motor delays.

Weeks after AD's diagnosis, I realized there were many medical publications about the ADNP gene but not of the actual syndrome. I learned that there was a neuroscientist in Israel, Professor Illana Gozes, who discovered the actual ADNP gene more than 15 years ago in her purposeful and tireless work in relations to Alzheimer's disease and autism. As a world-renowned neuroscientist, Professor Illana Gozes dedicates her life to developing ADNP-based treatments for patients afflicted with neurological degenerative diseases. I was the first parent to contact her. I told her we had families that needed her and in those moments, I felt she had nonverbally and verbally secured a commitment with understanding of our desperate cries for help. I could see a positive light in her and knew things would move forward with us. Again, the feeling of "alone" was withering away. We came to learn that ADNP is involved in many conditions - some of which are devastating neurodegenerative diseases such as Alzheimer's and ALS. Prof. Gozes gave us a glimmer of HOPE when she flew to the US to accept an award for RARE Champion of Hope for International Science Award promoted by our ADNP families (2016-https://globalgenes.org/press releases/2016-rare-champion-of-hope-honorees/). We realized she was taking a first and new step into pediatrics alongside us to help us maneuver a new way for our children. There seemed to be another feeling that if we continued to move forward together, the research might help all people, including the families and patients that suffer from other neurological disease that may receive a second chance at hope as well.

Patients with ADNP Syndrome, like AD, are mostly very young children. In the last years, however we have been fortunate to see that there are some young adults living with ADNP syndrome and some waited 16-22 years to get answers as well. With just over 200 cases of ADNP syndrome in the world (to date-https://www.adnpfoundation.org/ map.html) and after meeting a few mothers, we immediately could see how much our children both shared similar physical features, extreme likes and dislikes, sometimes the same medical experiences, similar genetic work-ups prior to ADNP diagnosis, and even same dental pattern which has become an early detection of the syndrome - discovered by one of the mothers (Sandra) herself, scientifically published in collaboration with Prof. Illana Gozes and much research work done in her lab (Gozes et al. 2018; Gozes et al. 2017a).

Seeing these families fully participating in the research and helping so many families understand the outcome through the years with $<400$ genes of the body, multiple body organs, and development all affected by ADNP, gives us hope. In the case of $\mathrm{AD}$, our daughter, she has a nonsense mutation at protein position Tyr719*, the most prevalent and known mutation of ADNP syndrome to date (Gozes et al. 2017b). This type of mutation in the DNA site creates a premature STOP codon, leading to the production of a shorter (truncated) ADNP protein, excluding protein regions important for ADNP protein activity. It was also documented in a medical journal in 2019 as having a more severe impact on the patients, when compared to other various mutations in the ADNP gene (Van Dijck et al. 2019). Interestingly, the expression for facial features is found in this area of the gene and that explains why so many of our children resemble one another maybe even look more like each other compared to their biological siblings.

Sadly, ADNP mutations affect multiple organs resulting in growth issues, rare speech disorders, and gastrointestinal impairments. Furthermore, some cases suffer from heart defects 
requiring open-heart surgeries. Often medical providers do not know enough about the condition to know how to treat the child from a multi-disciplinary specialty approach given the complexity, especially during illness. For us, speech is the highest loss of function given, obviously, basic verbal communication is most critical human need/function, followed by cognitive impairments leading to behavioral issues, which are often present in children who are verbally impaired. Just as a patient family observation, there seems to be a significant disparity between the receptive and the expressive language in the ADNP Kids currently observed which directly impacts their behavioral output. In AD's case, severe speech impairment leaves her at age 13 primarily nonverbal limiting her usage of approximated sign language, some basic verbal sounds and word approximations. Currently, AD is seeing two specialized speech therapists and is dependent upon an assistive technology (a device that speaks for her) to find her voice. Until more developmental studies are completed on more children in the US and abroad, we will not know if cognition is impacting speech, or whether this is a case of speech disorders (including severe apraxia/dysphasia, dysarthria, lack of coordinated speech) impacting cognitive behaviors.

MRI revealed AD has slight white matter loss of her cerebellum and her most recent 2018 MRI showed: "questionable T2 hyper intensity within the left hippocampus" which in itself is not a common finding in pediatrics according to two local neurologists. This finding is just a part of what other ADNP Kids are experiencing with these mutations - brain abnormalities and even cerebral atrophy has been noted and in some cases, risk for seizures/epilepsy. As a parent, knowing that other ADNP Kids MRI's have also presented abnormal findings (both present from birth and some changes that developed with time), it keeps me concerned that we must continue to study the complexity of the ADNP syndrome as a whole. It is important to treat the whole child - neuromuscular issues, orthopedic issues, complex speech disorders, frustrations over decreased communication and behavioral issues, and rightfully diagnose autism or autistic-like symptoms that many of the kids experience. Other diseases have both therapy and drug therapeutic interventions to help. Our ADNP kids are deserving of this kind of miracle too. For an explanation regarding the ADNP syndrome and/or list of the currently updated symptoms of this complex neurogenetic disorder, please visit the following websites: https://www.orpha.net/consor/cgi-bin/OC_Exp.php? Lng=EN\&Expert=404448; https://www.adnpkids.com/adnpkids-research-foundation.html.

With much sadness, we have lost three ADNP warriors and we pray knowing their lives served an amazing purpose for others to understand how ADNP can inflict the child and we strive that all ADNP Kids voices will be heard. That each of their unique stories will be told by their families so that the disease can be better defined, proper medical protocols can be set, insurances realize the need for intensive and aggressive interventions to battle regressions that can take place and so that all children with ADNP Syndrome and their families can live in HOPE.

We are "continuing in HOPE."

Prof. Gozes has developed a drug targeting our children (CP201; NAP) that could potentially help with brain connectivity, neuronal transport, and stop neural loss. CP201 serves almost as a gene therapy for ADNP Kids. It has been shown to increase vocalization, social behavior, and cognition in an ADNP-deficient animal model (Hacohen-Kleiman et al. 2018).

The drug CP201 for ADNP syndrome has been proven to be safe in humans in previous studies and has been recently approved the first ever therapeutic drug developmental path as orphan drug designation by the FDA (www.coronisns.com).

There is urgency with the need for continued research, for further development of treatments.

We are beyond grateful for Professor Illana Gozes and her team at Tel Aviv University for their tireless efforts in the laboratory to make the first clinical trial for CP201 drug come to life, aiming to treat all ADNP kids across the globe. To find out more about Professor Illana Gozes interesting work, go on her website: https://en-med.tau.ac.il/ profile/igozes.

Unfortunately, this requires massive funding.

Most people are unaware that some of the most known, yet rare diseases such as muscle dystrophy (MD) and cystic fibrosis (CF) are not government funded. ADNP is a Rare Disease in NEED of funding. The smaller the number of patient cases most often translates into less interest. All of us must CARE about RARE disease. Now is the time and need to fund the development for clinical trial use in the US.

We must find the funds to get the FIRST EVER TREATMENT off the ground. It is possible if we all work together to fund the source of our HOPE. If we spread the news and ask others for support. Think of how many medical miracles came in form of clinical trials and in some cases first drug treatments for other genetic diseases. We understand that a therapeutic for the ADNP gene also provides hope for other neurodegenerative diseases where loss of function is prevalent, e.g., ALS, Alzheimer's.

For more information on future activities toward better understanding of the syndrome, please visit (https://www. adnpfoundation.org/donatesupport.html). Please also check out Coronis Neurosciences webpage to read more about the development CP201 and meet the Coronis Neuroscience team (www.coronisns.com).

Acknowledgments We thank Mrs. Gal Hacohen Kleiman for her valuable input. Please also check a recent published magazine article: http:// www.charlestonphysicians.com/healthlinks/mayjune2019/76/. We are thankful for the contribution of the picture featuring two ADNP girls who share the same most prevalent mutation in ADNP meeting for the first time. One is $\mathrm{AD}$ and the second was described in a scientific publication featuring similarities to $\mathrm{AD}$ (Pescosolido et al. 2014). 


\section{References}

Gozes I, Van Dijck A, Hacohen-Kleiman G, Grigg I, Karmon G, Giladi E, Eger M, Gabet Y, Pasmanik-Chor M, Cappuyns E et al (2017a) Premature primary tooth eruption in cognitive/motor-delayed ADNP-mutated children. Transl Psychiatry 7:e1043

Gozes I, Patterson MC, Van Dijck A, Kooy RF, Peeden JN, Eichenberger JA, Zawacki-Downing A, Bedrosian-Sermone S (2017b) The eight and a half year journey of undiagnosed AD: gene sequencing and funding of advanced genetic testing has led to hope and new beginnings. Front Endocrinol (Lausanne) 8:107

Gozes I, Helsmoortel C, Vandeweyer G, Van der Aa N, Kooy F, Bedrosian-Sermone $\mathrm{S}$ (2018) Correction to: the compassionate side of neuroscience: Tony Sermone's undiagnosed genetic journeyADNP mutation. J Mol Neurosci 64:331

Hacohen-Kleiman G, Sragovich S, Karmon G, Gao AYL, Grigg I, Pasmanik-Chor M, Le A, Korenkova V, McKinney RA, Gozes I (2018) Activity-dependent neuroprotective protein deficiency models synaptic and developmental phenotypes of autism-like syndrome. J Clin Invest 128:4956-4969

Pescosolido MF, Schwede M, Johnson Harrison A, Schmidt M, Gamsiz ED, Chen WS, Donahue JP, Shur N, Jerskey BA, Phornphutkul C, Morrow EM (2014) Expansion of the clinical phenotype associated with mutations in activity-dependent neuroprotective protein. J Med Genet 51(9):587-589

Van Dijck A, Vulto-van Silfhout AT, Cappuyns E, van der Werf IM, Mancini GM, Tzschach A, Bernier R, Gozes I, Eichler EE, Romano C et al (2019) Clinical presentation of a complex neurodevelopmental disorder caused by mutations in ADNP. Biol Psychiatry 85:287-297

Publisher's Note Springer Nature remains neutral with regard to jurisdictional claims in published maps and institutional affiliations. 